(1)

CrossMark

\section{Supplemental oxygen and dypsnoea in interstitial lung disease: absence of evidence is not evidence of absence}

\author{
To the Editor:
}

It is with great interest that we read the recent systematic review by BeLL et al. [1] concerning the effects of oxygen therapy on dyspnoea and exercise capacity in patients with interstitial lung disease (ILD). The authors report that, while supplemental oxygen increases exercise capacity, it does not improve dyspnoea. Overall, this is a well-executed systematic review that accurately reflects the current literature; however, we believe the conclusion regarding the lack of benefit of supplemental oxygen on dyspnoea in ILD is misleading. This opinion is not based on the quality of the systematic review, but rather on the quality of the existing literature that was evaluated. We base our opinion on two important lines of evidence. First, previous studies that have evaluated the effect of supplemental oxygen on exertional dyspnoea in ILD patients only report peak or end-exercise dyspnoea ratings, thereby ignoring important clinically and physiologically relevant changes occurring at submaximal exercise intensities. Secondly, the exercise testing modalities employed are variable, such as self-paced walk tests [2-5] or incremental cycle exercise tests [6], which are often insensitive to changes in dyspnoea. An additional concern relates to the measures in place to reduce experimental bias, as the authors have addressed. Indeed, the only study included in their review that showed a beneficial effect of oxygen on dyspnoea did not have an appropriate control condition [5], making it impossible to rule out the placebo effect.

The measurement of dyspnoea is founded on the principle of psychophysics, whereby a stimulus is linked to a sensation [7]. It has been established that humans can reliably detect, quantify and discriminate qualitatively distinct sensations of dyspnoea provoked by different respiratory stimuli, either applied experimentally or as a result of disease [8]. Accordingly, the only way to properly evaluate the effects of any therapeutic intervention on exertional dyspnoea is to standardise the stimulus intensity. Specifically, this requires the assessment of dyspnoea at the same absolute exercise intensity and measurement time. It is extremely challenging to standardise exercise intensity during self-paced walk tests and thus, cycle exercise tests are more appropriate by design. Additionally, peak or end-exercise dyspnoea ratings are often insensitive to change following an intervention. This may be attributed to exercise tolerance improving as a result of the intervention and/or the fact that symptom-limited exercise cessation typically occurs at a similar level of dyspnoea for a given individual [9]. In other words, peak exercise is not the same stimulus if exercise time is increased (i.e. for incremental and constant work-rate cycle exercise tests) or if walking pace and/or distance changes (i.e. during self-paced walking tests).

In addition to standardising exercise intensity and measurement times, the magnitude of inspiratory oxygen fraction $\left(\mathrm{FIO}_{2}\right)$ delivered should be the same between individuals and should be of sufficient magnitude to reverse or, at the very least, cause a meaningful improvement in arterial oxygen saturation. With the exception of one study [6], all previous studies that examined dyspnoea in ILD used nasal cannulae or venturi masks to deliver the oxygen at various flow rates $[2-5,10]$. These gas delivery methods have well-established limitations $[11,12]$ and the effective $\mathrm{FIO}_{2}$ is likely to vary between individuals based on breathing pattern. These systems also have modest effects on improving arterial oxygen saturation compared with control conditions [2-5], which may explain, at least in part, previous negative studies of oxygen on dyspnoea in ILD. By contrast, administering oxygen through a reservoir bag connected to a two-way non-rebreathing valve, while less practical, allows for the precise control of $\mathrm{FIO}_{2}$ and is more effective at preventing arterial oxygen desaturation. This design is critical for testing the potential benefit of increased $\mathrm{FIO}_{2}$ on dyspnoea. If a benefit of supplemental oxygen during exercise is

@ERSpublications

Previous methodological flaws led to erroneous conclusions on the effects of oxygen on exertional dyspnoea in ILD http://ow.ly/Y48d30dCMk9

Cite this article as: Schaeffer MR, Molgat-Seon Y, Ryerson CJ, et al. Supplemental oxygen and dypsnoea in interstitial lung disease: absence of evidence is not evidence of absence. Eur Respir Rev 2017; 26: 170033 [https://doi.org/10.1183/16000617.0033-2017]. 

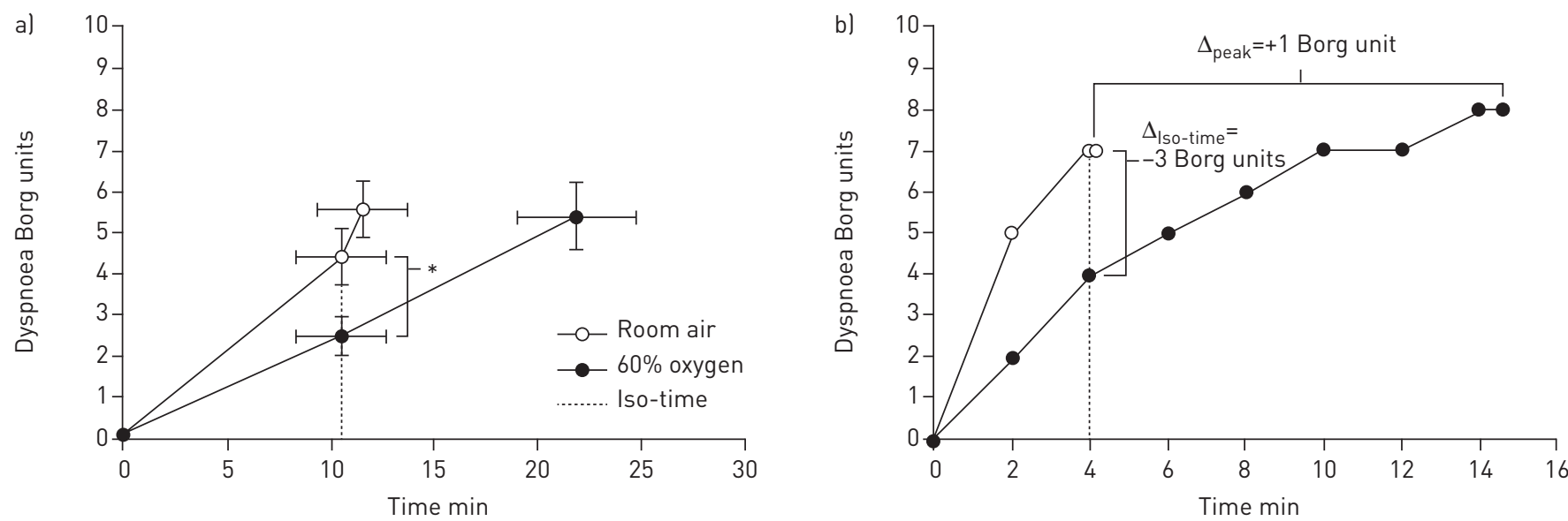

FIGURE 1 a) Dyspnoea response at rest, iso-time, and peak exercise in 20 patients with fibrotic interstitial lung disease during constant work-rate cycle exercise testing while breathing 60\% oxygen or room air. There was a statistically significant improvement in dyspnoea at iso-time but no change at peak exercise. Data are presented as mean \pm SEM. ${ }^{*}$ : $p<0.05$. b) Dyspnoea ratings every 2 min during constant load cycling in an individual patient while breathing $60 \%$ oxygen or room air. Dyspnoea ratings were reduced at all submaximal exercise times but the rating was increased at peak exercise. The Borg dyspnoea scale ranges from 0 to 10. Data from [13].

proven, there will be a need for additional research to identify the best means of translating these findings into clinical practice within a standard pulmonary rehabilitation setting. Thus, in order to make an unbiased evaluation of supplemental oxygen on dyspnoea, dyspnoea ratings should be measured at the same absolute submaximal work rate and exercise time (i.e. iso-time) while administering identical levels of effective $\mathrm{FIO}_{2}$ compared with an appropriate placebo condition.

We recently completed a study that addresses the aforementioned limitations in order to evaluate the effect of supplemental oxygen on dyspnoea during exercise in patients with ILD [13]. During this single-blind, randomised, placebo-controlled crossover study, 20 ILD patients performed two symptom-limited constant work-rate cycle exercise tests at $75 \%$ of peak work rate while breathing room air or supplemental oxygen $(60 \%$ oxygen $)$ in randomised order. Gas was delivered via a two-way non-rebreathing valve connected to a Douglas bag, such that the effective $\mathrm{FIO}_{2}$ was constant in all patients and was sufficient to reverse arterial oxygen desaturation, even at peak exercise (mean \pm SD arterial oxygen saturation measured by pulse oximetry was $98 \pm 1 \%$ with hyperoxia versus $90 \pm 4 \%$ with normoxia; $\mathrm{p}<0.001$ ). Gas cylinders and oxygen saturation monitors were obstructed from patient view, and gas delivery was identical for both experimental conditions. Briefly, dyspnoea was significantly reduced at iso-time with supplemental oxygen versus room air (mean \pm SD $2.5 \pm 2.1$ versus $4.4 \pm 3.1$ Borg units; $\mathrm{p}<0.01$ ), but was not different at peak exercise $(5.4 \pm 3.6$ versus $5.6 \pm 3.0$ Borg units; $\mathrm{p}=0.69)$ despite a significant improvement in exercise endurance time by an average of 10.3 min (mean \pm SD $21.9 \pm 12.9$ versus $11.6 \pm 10.0 \mathrm{~min} ; \mathrm{p}<0.001$ ). Figure 1 shows mean data as well as the dyspnoea response from an individual patient to illustrate the fundamental importance of measuring and reporting submaximal dyspnoea ratings. The data clearly show a substantial and clinically relevant reduction in dyspnoea at iso-time with hyperoxia in both examples, with no improvement at peak exercise. Relying exclusively on peak dyspnoea, as has been done in previous studies, results in the erroneous conclusion that supplemental oxygen is ineffective at reducing dyspnoea. The results of our study clearly demonstrate a reduction in dyspnoea with supplemental oxygen by an amount that well exceeds the minimal clinically important difference (derived from chronic obstructive pulmonary disease studies) of 1 Borg unit (scale of $0-10$ ) [14]. The positive results from this study formed the impetus for a large multicentre clinical trial evaluating the role of hyperoxia in a long-term exercise-training programme in patients with idiopathic pulmonary fibrosis [15].

The comprehensive systematic review by BeLL et al. [1] did not identify a clinically significant benefit of supplemental oxygen on dyspnoea in patients with ILD; however, this review is subject to the methodological limitations of the available literature. We believe that the primary conclusion to be drawn from the existing literature is that there is currently insufficient evidence to suggest either a benefit or a lack of benefit from supplemental oxygen during exercise in patients with ILD. Methodological factors, such as the mode of gas delivery, $\mathrm{FIO}_{2}$, the timing of dyspnoea measurements, and the exercise testing protocols, have been inadequately considered in most previous studies and probably contribute to the difference in conclusions between our study and the available literature. Before clinicians dismiss supplemental oxygen as a dyspnoea-relieving therapy in ILD, they should recognise that all studies reviewed by BELL et al. [1] were inadequately designed to evaluate the impact of supplemental oxygen on dyspnoea. 
Michele R. Schaeffer ${ }^{1,2}$, Yannick Molgat-Seon ${ }^{1,3}$, Christopher J. Ryerson ${ }^{1,4}$ and Jordan A. Guenette ${ }^{1,2,3}$

${ }^{1}$ Centre for Heart Lung Innovation, Providence Health Care Research Institute, University of British Columbia, St Paul's Hospital, Vancouver, BC, Canada. ${ }^{2}$ Dept of Physical Therapy, University of British Columbia, Vancouver, BC, Canada. ${ }^{3}$ School of Kinesiology, University of British Columbia, Vancouver, BC, Canada. ${ }^{4}$ Dept of Medicine, University of British Columbia, Vancouver, BC, Canada.

Correspondence: Jordan A. Guenette, Centre for Heart Lung Innovation, 166-1081 Burrard Street, Vancouver, BC, V6Z 1Y6, Canada. E-mail: jordan.guenette@hli.ubc.ca

Received: March 262017 | Accepted after revision: June 252017

Support statement: M.R. Schaeffer was supported by a fellowship from the University of British Columbia. Y. Molgat-Seon was supported by a fellowship from the University of British Columbia and a postgraduate scholarship from the Natural Sciences and Engineering Research Council of Canada. C.J. Ryerson was supported by a Scholar Award from the Michael Smith Foundation for Health Research (MSFHR). J.A. Guenette was supported by a Scholar Award from the MSFHR and a Clinical Rehabilitation New Investigator Award from the Canadian Institutes of Health Research. The funders had no role in the preparation of this correspondence.

Conflict of interest: Disclosures can be found alongside this article at err.ersjournals.com

Provenance: Submitted article, peer reviewed.

\section{References}

1 Bell EC, Cox NS, Goh N, et al. Oxygen therapy for interstitial lung disease: a systematic review. Eur Respir Rev 2017; 26: 160080.

2 Cao M, Wamboldt FS, Brown KK, et al. Supplemental oxygen users with pulmonary fibrosis perceive greater dyspnea than oxygen non-users. Multidiscip Respir Med 2015; $10: 37$.

3 Nishiyama $\mathrm{O}$, Miyajima $\mathrm{H}$, Fukai $\mathrm{Y}$, et al. Effect of ambulatory oxygen on exertional dyspnea in IPF patients without resting hypoxemia. Respir Med 2013; 107: 1241-1246.

4 Frank RC, Hicks S, Duck AM, et al. Ambulatory oxygen in idiopathic pulmonary fibrosis: of what benefit? Eur Respir J 2012; 40: 269-270.

5 Visca D, Montgomery A, de Lauretis A, et al. Ambulatory oxygen in interstitial lung disease. Eur Respir J 2011; 38: 987-990.

6 Harris-Eze AO, Sridhar G, Clemens RE, et al. Oxygen improves maximal exercise performance in interstitial lung disease. Am J Respir Crit Care Med 1994; 150: 1616-1622.

7 Zechman FW, Wiley RL. Afferent inputs to breathing: respiratory sensation. Compr Physiol 2011; 1: Suppl. 11, 449-474.

8 Parshall MB, Schwartzstein RM, Adams L, et al. An official American Thoracic Society statement: update on the mechanisms, assessment, and management of dyspnea. Am J Respir Crit Care Med 2012; 185: 435-452.

9 Puente-Maestu L, Palange P, Casaburi R, et al. Use of exercise testing in the evaluation of interventional efficacy: an official ERS statement. Eur Respir J 2016; 47: 429-460.

10 Dowman LM, McDonald CF, Bozinovski S, et al. Greater endurance capacity and improved dyspnoea with acute oxygen supplementation in idiopathic pulmonary fibrosis patients without resting hypoxaemia. Respirology 2017; 22: 957-964.

11 Markovitz GH, Colthurst J, Storer TW, et al. Effective inspired oxygen concentration measured via transtracheal and oral gas analysis. Respir Care 2010; 55: 453-459.

12 Maggiore SM, Idone FA, Vaschetto R, et al. Nasal high-flow versus Venturi mask oxygen therapy after extubation. Effects on oxygenation, comfort, and clinical outcome. Am J Respir Crit Care Med 2014; 190: 282-288.

13 Schaeffer MR, Ryerson CJ, Ramsook AH, et al. Effects of hyperoxia on dyspnoea and exercise endurance in fibrotic interstitial lung disease. Eur Respir J 2017; 49: 1602494.

14 Ries AL. Minimally clinically important difference for the UCSD Shortness of Breath Questionnaire, Borg Scale, and Visual Analog Scale. COPD 2005; 2: 105-110.

15 Ryerson CJ, Camp PG, Eves ND, et al. High oxygen delivery to preserve exercise capacity in patients with idiopathic pulmonary fibrosis treated with nintedanib. Methodology of the HOPE-IPF study. Ann Am Thorac Soc 2016; 13: 1640-1647.

Copyright @ERS 2017

ERR articles are open access and distributed under the terms of the Creative Commons Attribution Non-Commercial Licence 4.0

From the authors:

We would like to thank M.R. Schaeffer and colleagues for their correspondence regarding our recently published systematic review of oxygen for interstitial lung disease (ILD) [1]. In this review, we found no consistent evidence that oxygen therapy administered during exercise tests reduced the primary outcome of dyspnoea, although randomised crossover trials demonstrated improvements in exercise performance. We also reported that the quality of evidence was very poor, due to the retrospective nature of many studies, the potential for selection bias and lack of blinding.

We welcome the recent study by SCHAEFFER et al. [2], which adds important evidence regarding the acute effects of supplemental oxygen in ILD during laboratory testing. In a single-blinded randomised crossover study, the authors demonstrated that breathing oxygen at inspiratory oxygen fraction $\left(\mathrm{FIO}_{2}\right) 60 \%$ during a constant load cycle 
ergometer test resulted in decreased dyspnoea at iso-time (mean 1.9 Borg units), as well as a highly significant increase in endurance time (mean $10.3 \mathrm{~min}$ ). These results are consistent with our own recent findings in a double-blind randomised crossover study using $\mathrm{FIO}_{2} 50 \%$ during constant load cycling [3], where we found reduced dyspnoea at maximal workload (mean 1 Borg unit) and increased endurance time (mean $99 \mathrm{~s}$ ). Unfortunately, neither of these studies was available at the time our systematic search of the literature was conducted in March 2016, which illustrates a limitation of systematic reviews in an evolving field. We also acknowledge that we might have found greater improvements in our study [3] had dyspnoea been measured at iso-time, as suggested by the correspondents, although the improvement in dyspnoea at peak exercise is also an encouraging finding.

It is interesting to note impressive gains in exercise endurance time with supplemental oxygen in both studies $[2,3]$, which was consistent with our systematic review findings [1]. This is aligned with the patient experience of oxygen therapy. In our recent qualitative study [4], patients with ILD reported that, although they had expected oxygen therapy to reduce breathlessness, more common experiences included increased energy levels, better exercise capacity and enhanced physical activity. Unfortunately, there are no quantitative studies to aid our understanding of the impact of supplemental oxygen during daily life in people with ILD. Tightly controlled studies such as those of SCHAEFFER et al. [2] and our own [3] provide welcome hints of a possible symptomatic and physiological benefit in the laboratory setting, which could be relevant to the application of oxygen during exercise training in pulmonary rehabilitation, where oxygen delivery and exercise dose can be tightly controlled. However, the relevance of these findings to the use of ambulatory oxygen in daily life remains unclear. Both studies used high levels of inspired oxygen, which are impractical to deliver during free-living physical activity, and were conducted during cycling on an ergometer, an activity which is not representative of daily functioning. More evidence is urgently needed regarding the impact of ambulatory oxygen during day-to-day life on symptoms, physical activity and long-term outcomes in people with ILD.

We agree that the current body of evidence does not provide a definitive answer regarding the benefits (or otherwise) of oxygen therapy for people with ILD, particularly for oxygen therapy during daily life. Ongoing trials may provide useful direction $[5,6]$. However, we also note that use of oxygen therapy is not without costs to patients, who describe practical challenges, psychosocial impacts and unmet expectations [4]. Until robust data from clinical trials are available, we would encourage health professionals to actively engage individual patients in treatment decisions, providing the guidance needed to make informed decisions regarding the benefits and the costs of oxygen therapy during daily life.

@ERSpublications

Current evidence does not provide definitive answers regarding the benefits (or otherwise) of oxygen therapy for ILD http://ow.ly/4qr830dCYm4

Cite this article as: Bell EC, Cox NS, Goh N, et al. Supplemental oxygen and dypsnoea in interstitial lung disease: absence of evidence is not evidence of absence. Eur Respir Rev 2017; 26: 170072 [https://doi.org/ $10.1183 / 16000617.0072-2017]$.

Emily C. Bell ${ }^{1}$, Narelle S. Cox $^{2,3}$, Nicole Goh $^{3,4,5}$, Ian Glaspole ${ }^{5,6}$, Glen P. Westall ${ }^{5,6}$, Alice Watson ${ }^{2}$ and Anne E. Holland ${ }^{2,3,7}$

${ }^{1}$ Dept of Physiotherapy, Monash University, Clayton, Australia. ${ }^{2}$ Dept of Physiotherapy, La Trobe University, Melbourne, Australia. ${ }^{3}$ Institute for Breathing and Sleep, Melbourne, Australia. ${ }^{4}$ Respiratory and Sleep Medicine, Austin Health, Heidelberg, Australia. ${ }^{5}$ General Respiratory and Transplant, Alfred Health, Melbourne, Australia. ${ }^{6}$ Dept of Medicine, Monash University, Clayton, Australia. ${ }^{7}$ Dept of Physiotherapy, Alfred Health, Melbourne, Australia.

Correspondence: Anne E. Holland, La Trobe University Clinical School, Level 4, The Alfred Centre, 99 Commercial Rd, Melbourne, 3004, Australia. E-mail: a.holland@alfred.org.au

Received: June 232017 | Accepted: June 252017

Conflict of interest: Disclosures can be found alongside this article at err.ersjournals.com

Provenance: Submitted article, peer reviewed.

\section{References}

1 Bell EC, Cox NS, Goh N, et al. Oxygen therapy for interstitial lung disease: a systematic review. Eur Respir Rev 2017; 26: 160080.

2 Schaeffer MR, Ryerson CJ, Ramsook AH, et al. Effects of hyperoxia on dyspnoea and exercise endurance in fibrotic interstitial lung disease. Eur Respir J 2017; 49: 1602494.

3 Dowman LM, McDonald CF, Bozinovski S, et al. Greater endurance capacity and improved dyspnoea with acute oxygen supplementation in idiopathic pulmonary fibrosis patients without resting hypoxaemia. Respirology 2017; 22: 957-964.

4 Khor YH, Goh NSL, McDonald CF, et al. Oxygen therapy for interstitial lung disease. A mismatch between patient expectations and experiences. Ann Am Thorac Soc 2017; 14: 888-895. 
5 Ryerson CJ, Camp PG, Eves ND, et al. High oxygen delivery to preserve exercise capacity in patients with idiopathic pulmonary fibrosis treated with nintedanib. Methodology of the HOPE-IPF study. Ann Am Thorac Soc 2016; 13: 1640-1647.

6 Visca D, Tsipouri V, Mori L, et al. Ambulatory oxygen in fibrotic lung disease (AmbOx): study protocol for a randomised controlled trial. Trials 2017; 18: 201.

Copyright @ERS 2017

ERR articles are open access and distributed under the terms of the Creative Commons Attribution Non-Commercial Licence 4.0 